\title{
ANALYSIS AND APPLICATION OF LINEAMENTS EXTRACTION USING GF-1 SATELLITE IMAGES IN LOESS COVERED
}

\author{
Ling Han $^{1,2}$, Zhiheng Liu ${ }^{1, *}$ Zhongyang Zhao ${ }^{1}$, Yuming Ning ${ }^{1}$ \\ ${ }^{1}$ School of Geology Engineering and Geomatics, Changan University, Xian, P. R. China,710064 - hanling@chd.edu.cn \\ ${ }^{2}$ Shaanxi Key Laboratory of Land Consolidation and Rehabilitation, Chang' an University, Xian, P. R. China, 710064
}

Commission III, WG III/1

KEY WORDS: Geological lineaments, Remote Sensing, GF-1 satellite images, Hough Transform, Edge detection

\begin{abstract}
:
Faults, folds and other tectonics regions belong to the weak areas of geology, will form linear geomorphology as a result of erosion, which appears as lineaments on the earth surface. Lineaments control the distribution of regional formation, groundwater, and geothermal, etc., so it is an important indicator for the evaluation of the strength and stability of the geological structure. The current algorithms mostly are artificial visual interpretation and computer semi-automatic extraction, not only time-consuming, but labour-intensive. It is difficult to guarantee the accuracy due to the dependence on the expert's knowledge, experience, and the computer hardware and software. Therefore, an integrated algorithm is proposed based on the GF-1 satellite image data, taking the loess area in the northern part of Jinlinghe basin as an example. Firstly, the best bands with 4-3-2 composition is chosen using optimum index factor (OIF). Secondly, line edge is highlighted by Gaussian high-pass filter and tensor voting. Finally, the Hough Transform is used to detect the geologic lineaments. Thematic maps of geological structure in this area are mapped through the extraction of lineaments. The experimental results show that, influenced by the northern margin of Qinling Mountains and the declined Weihe Basin, the lineaments are mostly distributed over the terrain lines, and mainly in the NW, NE, NNE, and ENE directions. It provided a reliable basis for analysing tectonic stress trend because of the agreement with the existing regional geological survey. The algorithm is more practical and has higher robustness, less disturbed by human factors.
\end{abstract}

\section{INTRODUCTION}

Geological structure is the deformation or displacement of rock stratum or rock mass under the action of internal and external stresses of the earth. The study of structure brings convenience for oil exploration, groundwater storage, understanding the mechanisms of environmental disasters, for instance, earthquake, flood and landslides (Marghany and Hashim, 2010). Lineaments may reflect discontinuities of surfaces of in the rock$\mathrm{s}$ or may reflect geological structures, topographic features or human-made features (Qari, 2011). A number of experts extracted and interpreted from satellite images and digital elevation models (DEMs), and provided an effective scientific basis for studying the trend of regional plate movement and tectonic framework (Masoud and Koike, 2011, Ahmadirouhani et al., 2017). However, the extraction and analysis of geological lineaments have always been a time-consuming and laborious task, because geological experts usually use the traditional method of visual interpretation and semi-automatic interpretation (Kusk and Krbcov, 2015). Semi-automatic methods are mainly based on the images filtering, band math, or edge enhancement such as Hough Transform (Soto-Pinto et al., 2013), Segment Tracing Algorithm (Koike et al., 1995), etc. All those algorithms are more dependent on the processing abilities of the computer, images size and resolution, and the types of the regional land-use. These methods have been implemented in some place where bedrock cutting is evident, and we can easily find the strike ridges and dykes, only few experts pay attention to the special geomorphological areas where the lineaments covered by deep vegetation and soil, so as

*Corresponding author: School of Geology Engineering and Geomatics, Changan University, Xian, P. R. China, 710064- liuzhiheng@chd.edu.cn to joints and faults are negative to detect (Hashim et al., 2013). In this unique region, not only the area is difficult to get into and investigate, but also the traditional method cannot be used owing to the inconspicuous feature of the lineaments. In view of these two points, we focus on the main research questions:

- What are the linear features of geological structures in remote sensing images?

- How to enhance the linear feature in the satellite images?

- How to extract rapidly in spatial geomorphological area and guarantee high accuracy?

The above research questions underline the need for a new resolution to deal rapidly with the problem of the extraction in the special place. Consequently, a lineaments extraction algorithm proposed in this study, which taking a loess area in the northern part of Jinlinghe basin as an example, and based on following 3 steps. First, the best bands of the image was selected by optimum index factor (OIF), and the redundant bands were removed. Secondly, line edge was highlighted by using Gaussian high-pass filtering and tensor voting. Finally, the Hough Transform was applied to turn the shape of the geological lineaments in the plane coordinate system into the statistical peak problem in the polar coordinate system to detect the geological lineaments.

In the experiments of this study, data set from GF-1 satellite images was used to test the performance of our proposed method. In the test of the density and orientation analysis, almost all extracted lineaments can be recognized clearly after the processing of linking end-points by the orientation and length, and have high 
consistence with regional geological survey results. However, it is not easy to improve the speed of computer processing. In addition, more land-use boundaries identified as lineaments after filtering can be suppressed, which increase the accuracy of the results. Furthermore, the orientations and density of linear structures are also important indicators of tectonic stress intensity. In this case, the direction and intensity of structures in the study area will be further analysed.

\section{STUDY AREA AND DATA}

\subsection{Study Area}

The study area is located in the northern Baoji, which covered very thick loess on the surface (Figure 1a). Its geographical range is $34^{\circ} 23^{\prime} 12^{\prime \prime}-34^{\circ} 35^{\prime} 12^{\prime \prime} \mathrm{N}$, and $106^{\circ} 59^{\prime} 3^{\prime \prime}-107^{\circ} 10^{\prime} 16^{\prime \prime} \mathrm{E}$, and covers about $382.8 \mathrm{~km}^{2}$. Under the influence of the new activity of large regional fault, the mountain area is uplifted with the local result of relative declined at the same time, and the fault basin is formed. Jinlinghe Basin is the southernmost part of the northern segment of the north-south tectonic belt in China, and is the transition zone between the east-west and south-north mega structures of China mainland (Figure 1b). The main structures in the study area are northwest faults, and the southern area is complicated Qinling fold belt. From the results of investigation, Taoyuan-Guichuansi Fault $\left(\mathrm{F}_{1}\right)$ and Guguan-Guozhen Fault $\left(\mathrm{F}_{2}\right)$ are buried active faults, which have strong extrusion thrust properties and horizontal left-hand motion. Taoyuan-Guichuansi Fault $\left(\mathrm{F}_{1}\right)$ and Qinling Piedmont Fault $\left(\mathrm{F}_{5}\right)$ divide the area into 3 regions, which are the weak uplifting area of Liupan-Longshan Basin $\left(\mathrm{I}_{1}\right)$, the uplifting area of Qinling Mountains $\left(\mathrm{I}_{2}\right)$ and the descending area of Qianhe Basin $\left(\mathrm{I}_{3}\right)$, respectively. However, it is difficult to find out the obvious fault point in field investigation due to the thick Quarternary Loess. Therefore, the new resolution to extract lineaments in this special geomorphologic region turns to be imperative.

\subsection{Data Collections}

GF-1 satellite was launched in April 2013, followed by GF-2 on August 2014, GF-3 on August 2016 and GF-4 on December 2015. GF-1 satellite have 2 sensors, PMS and WFV, respectively. PMS have higher spectral resolution, which offer linear enhanced capabilities (Table 1). However, most experts paid more attention on the extraction of traditional land features, such as water boundaries, cloud and suspended particulate matter, etc., (Li et al., 2015, Du et al., 2017, Li et al., 2017). GF-1 satellite image provides rich linear features, which are better for understanding the tectonic movement and fracture orientation in this area, and can be used for lineament interpretation. There are few researchers studied on regional geological investigation by using visual interpretation, it is a pretty waste of data resources. In view of this, GF-1 PMS product used for Jinlinghe Basin in this study was acquired on May 27, 2015. The values of image path/row, cloud covered, solar azimuth, and solar zenith are $14 / 98,0,148.53$, and 74.8854 , respectively. It can be radiation calibrated, atmosphere corrected, image merged and clipped by using the parameter provided by www.cresda.com. In this research, the image was pre-processing for getting a better image under the help of ENVI version 5.3.1, MATLAB version 2014a, and ArcGIS version 10.2 software packages.
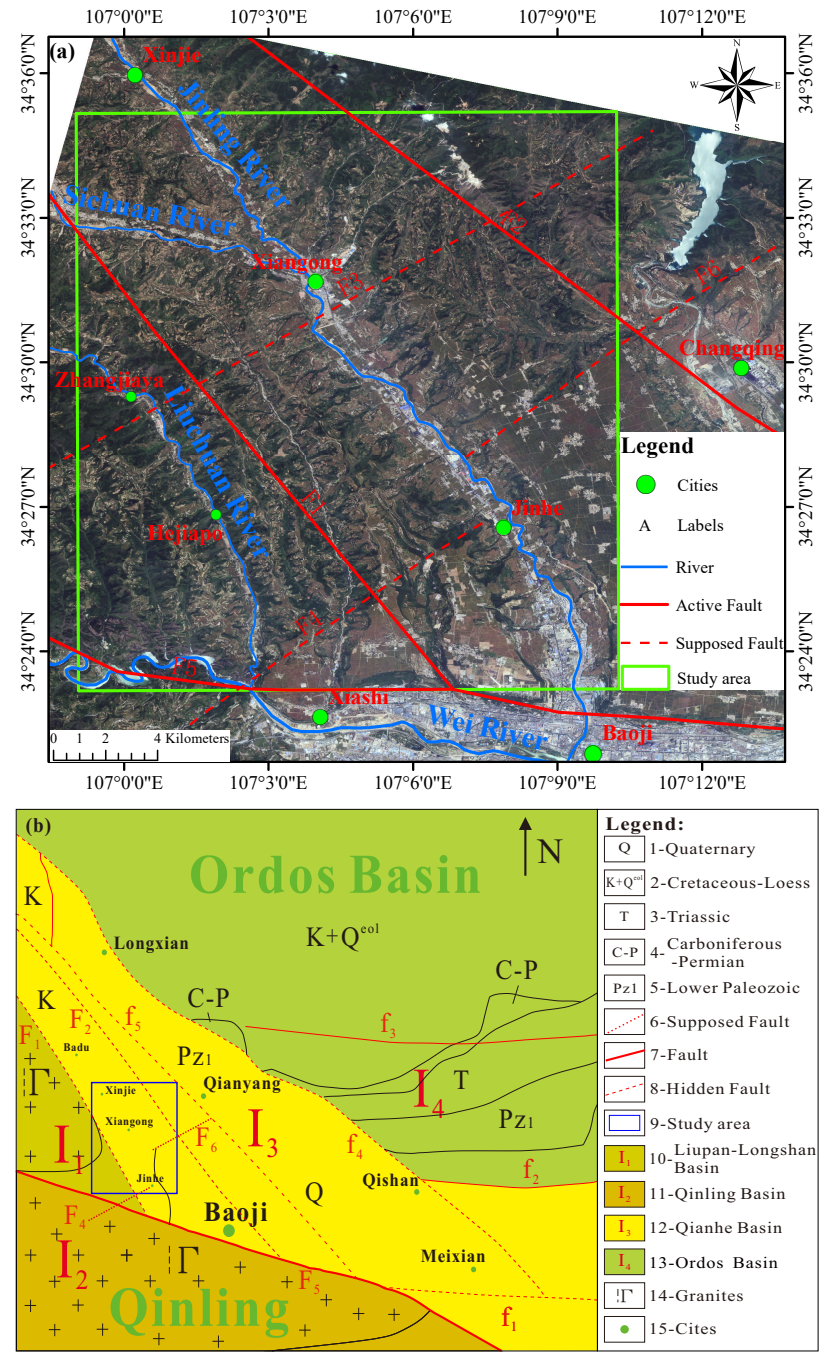

Figure 1. Study area and data collections (a) GF-1 satellite image; (b) Regional structure background (modified from China Earthquake Administration, 1988)

\section{METHODOLOGY}

\subsection{Data Pre-processing}

Data pre-processing has been proven to enhance the linear feature and increase the quality of interpretation. Optimum Index Factor (OIF) is one of the useful methods in the region of image enhancement, and is used in this study to get the best bands composition for lineaments extraction. The character of the OIF is that it takes advantage of the correlation between bands. Many research have shown that the greater the correlation coefficient, the more redundant information between bands so that we cannot highlight colour and distinguish information of different geological bodies. To achieve the purpose of enhancement, the first aim is to count the correlation coefficients and acquire the biggest OIF in GF-1 images. The OIF can be written as follows:

$$
O I F=\frac{\sum_{i=1}^{n} S_{i}}{\sum_{i=1}^{n} \sum_{j=1}^{n}\left|R_{i j}\right|}
$$

Where $S_{i}$ is the standard deviation of the $i_{t h}$ band; $R_{i j}$ is the 


\begin{tabular}{|c|c|c|c|c|}
\hline Sensors & Spectrum & $\begin{array}{l}\text { Band } \\
\text { order }\end{array}$ & $\begin{array}{c}\text { Wavelength } \\
(\mathrm{nm})\end{array}$ & $\begin{array}{l}\text { Resolution } \\
\text { (m) }\end{array}$ \\
\hline \multirow{5}{*}{ PMS } & Panchromatic & 1 & $450-900$ & 2 \\
\hline & Blue & 2 & $450-520$ & \multirow{4}{*}{8} \\
\hline & Green & 3 & $520-590$ & \\
\hline & Red & 4 & $630-690$ & \\
\hline & Nir & 5 & $770-890$ & \\
\hline \multirow{4}{*}{ WFV } & Blue & 6 & $450-520$ & \multirow{4}{*}{16} \\
\hline & Green & 7 & $520-590$ & \\
\hline & Red & 8 & $630-690$ & \\
\hline & Nir & 9 & $770-890$ & \\
\hline
\end{tabular}

Table 1. Characteristics of GF-1 PMS Satellite Image (data from http://www.cresda.com)

correlation coefficient between the $i_{t h}$ and $j_{t h}$ bands.

\subsection{Edge Detection}

The removal of redundant bands is just one step in the lineament extraction due to the exist of linear noise in the image, such as land-use boundaries, roads, rivers, etc. Therefore, to minimize noise in GF-1 image, the Gaussian high-pass filter is known to be a simple and useful way. Because it suppresses the low frequency signal of the image spectrum while preserving the high frequency signal. The high frequency part in the frequency domain corresponds to the place where the gray scale changes sharply in the image. These places are often the edges of lineaments and better for the extraction. So we applied the Gaussian high-pass filter in this study, and it can be written as follows:

$$
H(u, v)=1-e^{-\frac{D^{2}(u, v)}{2 D_{0}^{2}}}
$$

Where $\boldsymbol{D}(\boldsymbol{u}, \boldsymbol{v})$ is the distance between the center $(u, v)$ of the frequency domain and the center of the frequency rectangle; and $\boldsymbol{D}_{0}$ is a cut-off frequency.

The next step is edge detection. A great deal algorithm has been accomplished in recent years. The commonly used detector is Canny, Robert, Sobel, Prewitt, and LOG. The location of Robert operator is accurate, but it is sensitive to noise for lacking in smoothness. Both the Prewitt and Sobel operator are first order derivative, the processing effect is not ideal for the image with mixed and complex noise. In the method of Log filter, a second order derivative, the greater the smoothing effect is, the better the noise removal is, which result in the greater the loss of the details of the image and a low edge accuracy. Therefore, there is a contradiction between edge location accuracy and noise elimination level, which should be properly selected according to the specific problem of noise level and edge location accuracy. Actually, single response to edge points, the ability of localization, and the advancing adaptability made Canny detector get more experts concern. The edge extracted by using Canny operator is really clear and has good closure, so it is not easy to be affected by errors. However, Canny operator also has some limitations: there is false edge phenomenon in edge detection, which affects the results authenticity. So it can be eliminated manually if the false edge is small. More false edges will appear while the gray scale mutation is large, especially in the areas with dense ridges, and the method will be difficult to obtain ideal results.

Therefore, concerning the characteristics of the above operators, we use a new method of edge detection, which proposed by a team led by Grard Medioni at the end of the last century and applied effectively in computer graphics. The filter image is turned to be a binary image, and its characteristics can be represented by eigenvalues and eigenvectors. The tensor can stand for these information of points, so we turned the image points into tensor. We can use the $\boldsymbol{O}$ and $\boldsymbol{E}$ represent points where pixels are zero and non-zero, respectively. The image data with linear geometric features are included in the voting process, and the mutual transfer of information between data and data is realized through different voting fields. All the points are voted by the surrounding pixels, the points on the linear edge getting a non-zero vector sum due to the transformed voting information, but the non-linear points acquired the opposite results. So the linear edge is preserved and the non-edge is removed. The voting is directional, and each pixel is voted by the neighbour to obtain the final result. The schematic diagram of voting can be shown as Figure 2, and we calculate the voting value as follows:

$$
V=D F \cdot N N^{T}
$$

where $\boldsymbol{N} \boldsymbol{N}^{T}$ is the gradient, and $\boldsymbol{D F}$ is Significant attenuation function, it can be calculated as follows:

$$
\begin{gathered}
D F=e^{-\frac{\left(L^{2}+c k^{2}\right)}{\sigma^{2}}} \\
c=\frac{-16(\sigma-1) \log (0.1)}{\pi^{2}}
\end{gathered}
$$

where $\boldsymbol{L}$ is the length of the curve; $\boldsymbol{k}$ is the curvature; $\sigma$ is the region of voting neighbourhood; $\boldsymbol{c}$ is the coefficient controlling the degree of curvature attenuation.

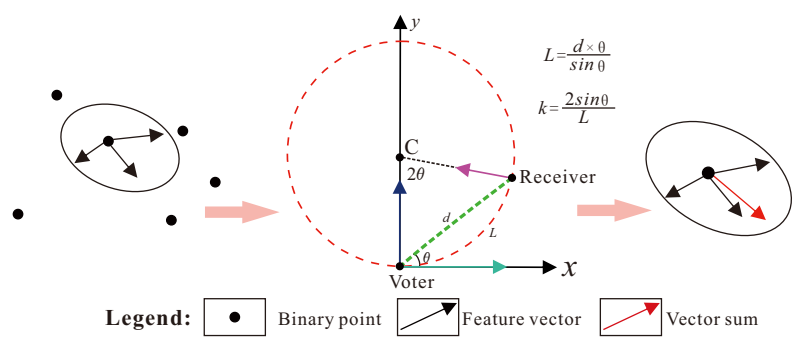

Figure 2. The schematic diagram of voting process

Therefore, we can get the cumulative result $\boldsymbol{T}$ of points, and decompose with Equation (6).

$$
\begin{aligned}
& T=\left[\begin{array}{ll}
\overrightarrow{e_{1}} & \overrightarrow{e_{2}}
\end{array}\right]\left[\begin{array}{cc}
\lambda_{1} & 0 \\
0 & \lambda_{2}
\end{array}\right]\left[\begin{array}{l}
\overrightarrow{e_{1}} \\
\overrightarrow{e_{2}}
\end{array}\right] \\
& =\lambda_{1} \overrightarrow{e_{1}}{\overrightarrow{e_{1}}}^{T}+\lambda_{2}{\overrightarrow{e_{2}}}_{{\overrightarrow{e_{2}}}^{T}}^{T} \\
& =\left(\lambda_{1}-\lambda_{2}\right){\overrightarrow{e_{1}}}_{\vec{e}_{1}^{T}}^{T}+\lambda_{2}\left(\overrightarrow{e_{1}}{\overrightarrow{e_{1}}}^{T}+{\overrightarrow{e_{2}}}_{\vec{e}_{2}}^{T}\right)
\end{aligned}
$$

where $\overrightarrow{e_{1}}$ and $\overrightarrow{e_{2}}$ denote the normal and tangent directions of the point, respectively, and $\lambda_{1}$ and $\lambda_{2}$ denote the size of them. So the problem turns to be the solution of $\lambda_{1}$ and $\lambda_{2}$ : (1) $\left(\lambda_{1}-\lambda_{2}\right)>$ $\lambda_{2}$, the pixel is a point located on the linear edge; (2) $\lambda_{1} \approx \lambda_{2}$, the pixel is located in the inner area or at the crossing-point of a region and is judged to be a non-edge point. 


\subsection{Lineaments Extraction}

In this study, we implemented the Hough transform to obtain the final lineaments map from the binary image produced by the Tensor voting. For the ability of locating and identifying for the lineaments, HT was regarded as an effective and common numerical algorithm, and had been widely used in this domain. Every binary image has the special linear feature for the ground truth, both the two edge can be linked to a line according to the orientation and spacing. In this method, each point in figure space $\left(x_{i}, y_{i}\right)$ can be turned to be a straight line in parameter space $(\mathrm{m}, \mathrm{c})$, which can be written as follows:

$$
y_{i}=m x_{i}+c
$$

Both two points in a same line can be transformed to two lines in the parameter space $(\mathrm{m}-\mathrm{c})$. Therefore, all points can cross the same point parameter space. Considering the value of $\mathrm{m}$ and $\mathrm{c}$ may be too large, Duda and Hart (1972) introduced polar coordinate method of lines, defined by $(\rho, \theta)$ of the normal vector to the line (Duda and Hart, 1972), which can be shown as follows:

$$
\rho=x \cos \theta+y \sin \theta
$$

Finally, the points on the linear edge are made to a point in the parameter space because of the consistency of the edge properties. So it converts the problem of point detection from the image space into peaks detecting in the parameter space, which can be drawn as Figure 3.

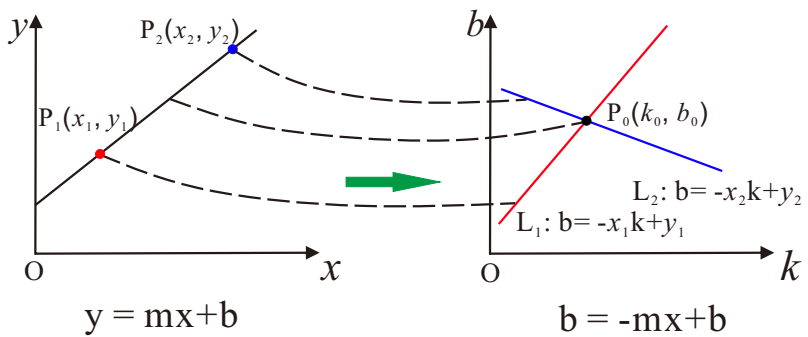

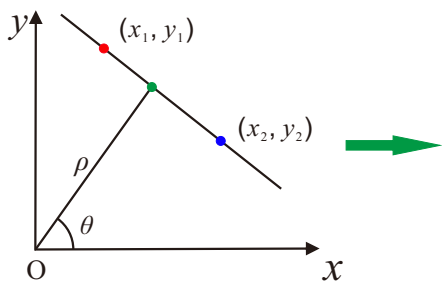

$\mathrm{y}=\mathrm{mx}+\mathrm{b}$

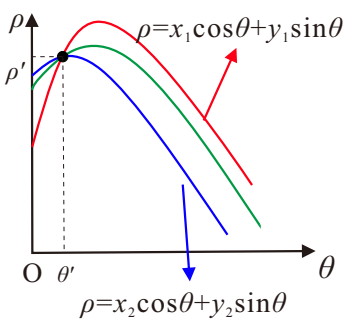

$\rho=x_{2} \cos \theta+y_{2} \sin \theta$

Parameter space

Figure 3. The flow chart of the Hough Transform

\section{RESULTS AND DISCUSSION}

\subsection{Lineaments Extraction Results}

The integrated method was implemented in the northern Baoji loess area. Firstly, we counted the correlation coefficient matrix of the images in Table 2. The results showed that Band 4 had a

\begin{tabular}{|c|c|c|c|c|}
\hline $\mathrm{R}_{i j}$ & Band1 & Band2 & Band3 & Band4 \\
\hline Band1 & 1.00000 & 0.96179 & 0.92295 & -0.30266 \\
Band2 & 0.96179 & 1.00000 & 0.96239 & -0.24780 \\
Band3 & 0.92295 & 0.96239 & 1.00000 & -0.31790 \\
Band4 & -0.30266 & -0.24780 & -0.31790 & 1.00000 \\
\hline
\end{tabular}

Table 2. Correlation coefficient matrix of GF-1 satellite images bands in the study area

low correlation coefficient with the other bands, so band 4 was the preferred band to be synthesized with other bands. The standard deviation for each band were 37.044, 47.074, 60.798 and 72.558, respectively. Then the OIF was calculated, and the values of 32-1, 4-2-1, 4-3-1, 4-3-2 obtained were 50.899, 103.604, 110.398 and 118.076, respectively. It could be seen that the best form of band synthesis with largest OIF was 4-3-2, which enhanced the linear edges and removed the redundant bands, especially, highlighted some of the less obvious linear features. Therefore, the experimental image was assigned to Band 4, Band 3, and Band 2.

Considering the processing ability of MATLAB 2014a, the GF-1 satellite image of the study area was divided into 9 sub-images, and sequentially numbered (a)-(i) from top to bottom, left to right. Taking the second, fourth and eighth blocks as examples, the results of Gaussian high-pass filter, tensor voting, and final lineaments were mapped as Figure. 4.

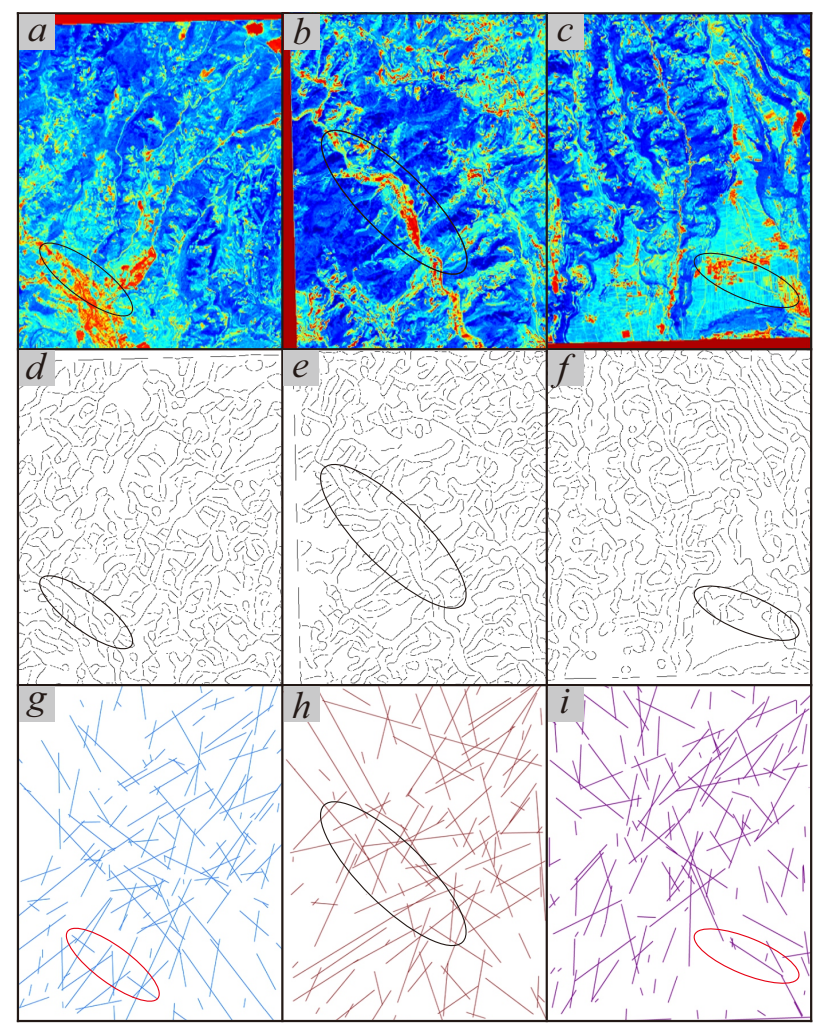

Figure 4. The lineaments extraction example: the first row is the Gaussian high-pass results, the second row is the edge detection by tensor voting, and the third row is the lineaments after Hough transform

Gaussian high-pass filter allowed the region where the gray frequency changes fast to passes through, but suppresses the opposite region. This feature is characterized by rapid gray scale changes and high gradients, which made edge points more clear and easily to detect in the image. The more obvious the degree 


\begin{tabular}{|c|c|c|c|c|}
\hline Degree & length class & $\begin{array}{c}\text { Length } \\
(\mathrm{m})\end{array}$ & Number & $\begin{array}{c}\text { Percent } \\
(\%)\end{array}$ \\
\hline 1 & Very short & $0.5-1.1$ & 346 & 58.85 \\
2 & Short & $1.1-1.7$ & 169 & 28.74 \\
3 & Medium & $1.7-2.3$ & 57 & 9.69 \\
4 & Long & $2.3-2.9$ & 12 & 2.04 \\
5 & Very long & $>2.9$ & 4 & 0.68 \\
\hline
\end{tabular}

Table 3. Lineaments statistics

of rock fragmentation, the denser the lineaments at the surface. The first row of Figure 4 involves the mountain and flat areas in the countryside. The critical part often has a large gray scale mutation, so the obvious color difference is found on the filtered image. The images were enhanced by tensor voting, especially in places where the slope change was small. Computing the peaks in parameter space using of HT shortened the time and got the satisfactory results. It was worth noting that in the vicinity of Jinlinghe, because of the complex geomorphology and large topographic fluctuation in the study area, residential areas were often arbitrarily constructed and had no regular geometric shapes, resulting in a small amount of noise as shown in Figure. 4 a, c. But the results showed that the existence of a small amount of noise had no effect on the interpretation of the large structure of the region. Such noise is usually short in length, so it can be eliminated by threshold elimination only in the high-precision interpretation of the lineaments. Similarly, there was directional consistency in the gray scale transformation controlled by the structure, while the artificial ditch was not controlled by the structure and lacked directivity, so the lineaments around the former were preserved, and the latter was removed. Due to the ridge lines controlled by the structure, so the results of the lineaments extraction could indicate the directions and the stress. Actually, the lineaments extracted by using Hough transform was attached to the ridge line in the image, but the density was high, which further revealed that the squeezing degree of Liupan-Longshan was increasing. The final lineaments distributions are shown in Figure 5. Total numbers of 1262 lineaments are detected and grouped into 588 lineaments in the GF-1 satellite images, and the final lineaments output are shown in Table 3. The area with the densest lineaments was located in the three sub-blocks of Figure 4, which was related to the landform uplift and the lithology of the bottom. The LiupanLongshan orogenic belt compressed eastward, the Qianhe basin was declined in difference, and the northern margin of Qinling uplifted, so the whole state of the study area was dense west and sparse east. From Table 3, the lineaments length of the study area was very short, concentrated between $0.5-1.1 \mathrm{~km}$ and related to the covered loess on the surface.

\subsection{Density Analysis}

In order to study the existence of lineaments distribution and hidden structure in this study area, it is a practical method to analyse the density of the extracted lineaments. The spatial distribution of lineaments is reflectance of the structure on the surface. The denser the linear structure, the greater the density and the degree of rock fragmentation controlled by the structure. Although the surface loess is thicker and the rocks are not exposed obviously, the linear structure is consistent in the local control direction, so the direction of the structure can be inferred from the degree of density accumulation. As shown in Figure 6, lineaments were concentrated in sub-maps b, c, d and h. The bedrock was seriously fractured, accompanied by landslides and mudslides, which was the critical part of the basin and mountain area, and the crossing of structure. At the same time, because of its red sandstone

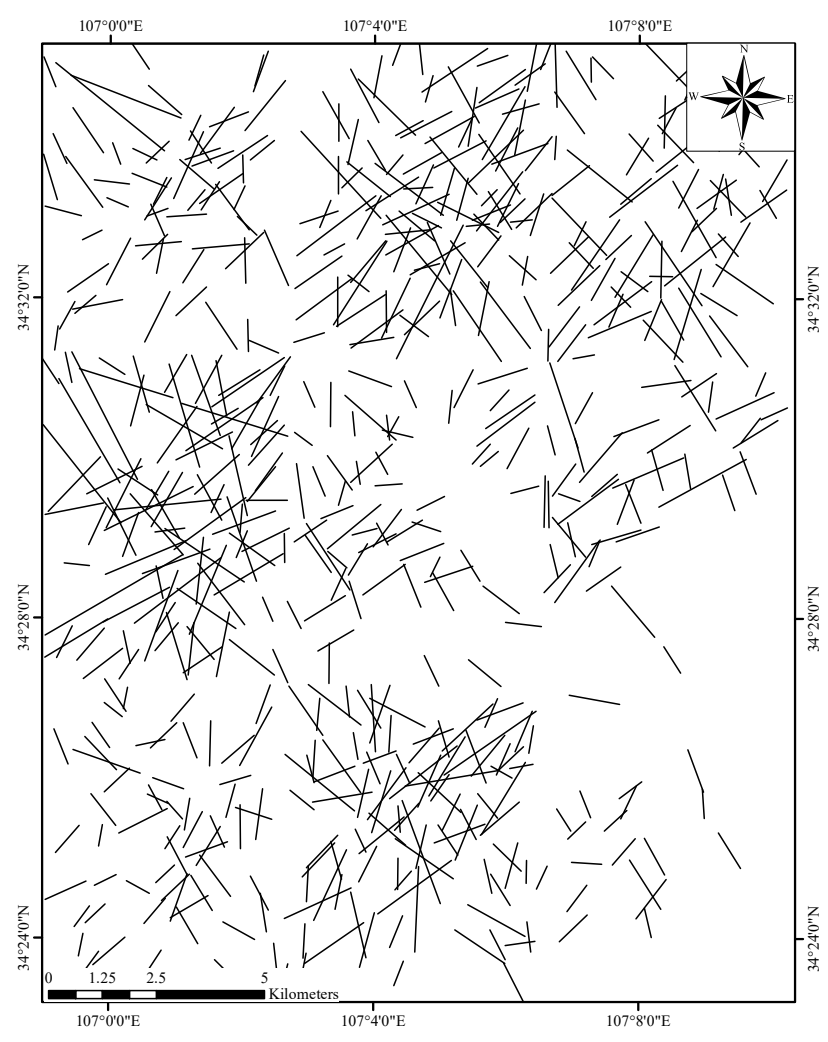

Figure 5. The final lineaments

and siltstone composition, the region had poor water content. It was easy to scour with the flood, forming a large number of vertical loess slope walls, so it further indicated that the tectonic environment was complicated in this area. The analysis results of lineament density showed that the lineaments were concentrated in the western region with high degree of fragmentation and density, and the compression gradually extended eastward, accompanied by local uplift and strike-slip movement. Because most of the faults are hidden faults, it was difficult to find obvious fault feature points on the surface of the earth. However, it provided a powerful basis for analysing the location of hidden faults. Furthermore, the results also indicated the main directions of the structure, the predominant direction were NNE (direction of azimuth angle $0-30^{\circ}$ ), NE (direction of azimuth angle $30-60^{\circ}$ ), NW (direction of azimuth angle $310-330^{\circ}$ ), and the second dominant directions were NNW and WNW. The results obtained in this research agreed well with the regional trends and the local geological maps.

\subsection{Orientation Analysis}

Furthermore, the azimuths of the lineaments in each sub-images were measured and plotted as rose diagrams. As shown in Figure 7 , the lineaments orientations were well mapped in the study area. The rose diagrams showed four prominent trends: NWSE, NE-SW, NNE-SSW, and ENE-WSW, arranged by 10 degrees clockwise. Lineament directionality for these sub-areas showed a statistical distribution similar to the total fault stress, especially the active faults. The southern part of the study area was located at the junction of the large regional faults (e.g. Figure 7-a, d, e, $\mathrm{g}$ and $\mathrm{h}$ ), and the NW and NE fault interlaced aggregation, so the linear structure directions were more dispersed. While the subimages (e.g. Figure 7-c, f, and i) near the Qianhe-Weihe basin were low in topography and stable, the linear structure directions were more concentrated and clear, which clearly showed the di- 


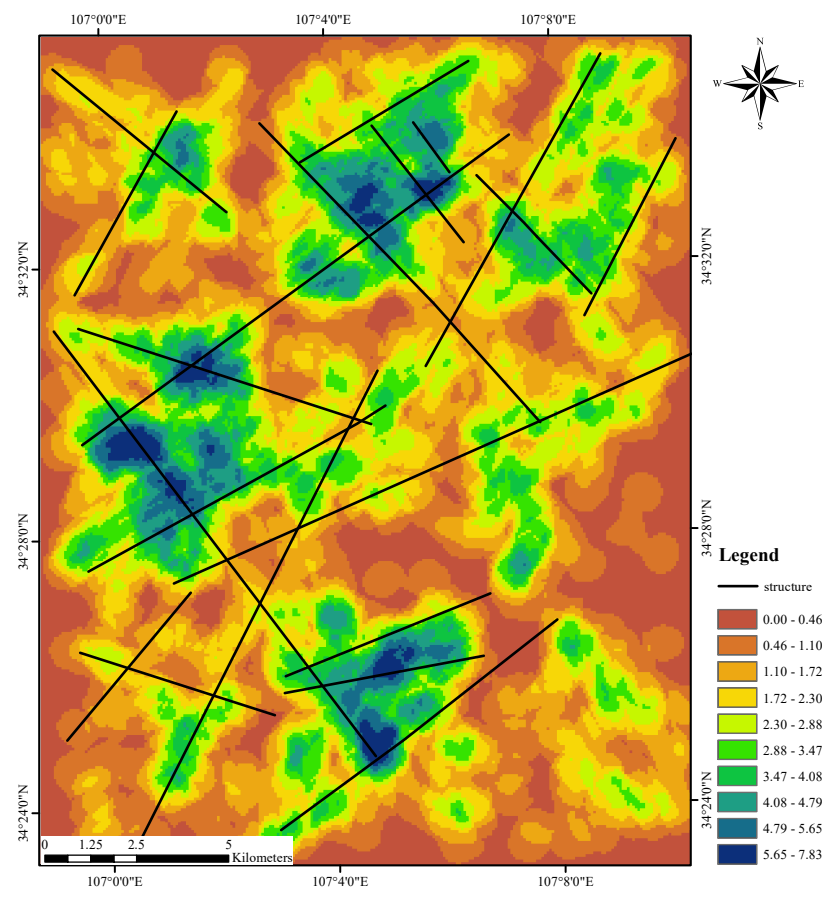

Figure 6. Lineaments density map

rections of principal stress of NE hidden fault. In addition, the orientation of the lineaments may indicate the trend of the fault movement. Under the influence of the new activities of large regional faults, the mountains in the study area were uplifted in a large area and squeezed towards the northeast, and the QianheWeihe Basin in the northeast of the study area had declined unevenly for a long time. The formation of ridge and valley lines in this area was closely related to the direction of extrusion. The whole ridge and valley lines extended in the NE and NW directions, with the tendency of local diffusion to the surrounding direction. The complex movement trend led to the frequent occurrence of earthquakes in this area, and the controlling feature by the hidden faults were more obviously. In the study area, the NE fault showed the characteristics of linear structural concentration and extension direction change, which reflected the area located in the fault zone. While the northwest side showed the discontinuous feature, which was obviously cut by the north-east fault, and is in accordance with the actual situation.

\section{CONCLUSION}

This study presented the lineaments extraction results of GF1 satellite images conducted in the Jinlinghe Basin, located in northern Baoji loess covered area. The main aim is to enhance the linear feature and decrease the effect of the noise. Considering the thick loess in this unique area, an integrated method for lineaments in extraction was implemented, and provided a reliable and powerful basis for revealing the tectonic movement in this area. The best bands composition (4-3-2) was firstly chosen by OIF. The redundant bands were removed, which were good for noise reduction in the subsequent process. Taking advantage of the tensor voting to edge detection, the edges of linear features in GF-1 image were enhanced. Compared with other traditional methods to edge detection, tensor voting is superior to the other algorithms in terms of de-noising ability and complete representation ability of linear, so the lineaments enhancement turned to be easily. In particular, the ridge lines controlled by the structure were highlighted and the noise caused by the artificial ditch

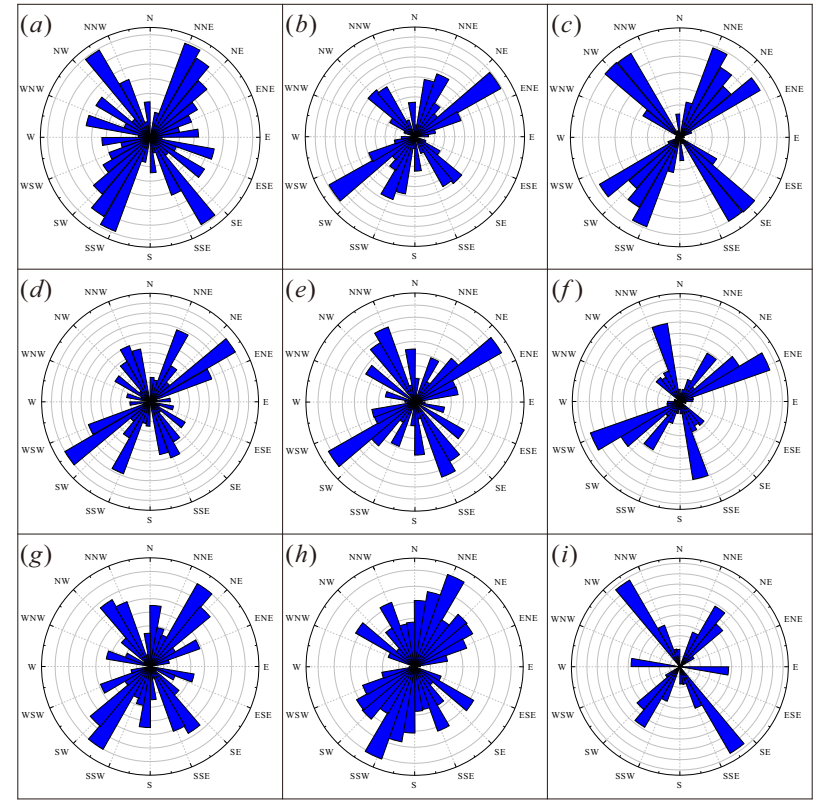

Figure 7. The main orientation of the lineaments for each sub-images

were suppressed. Hough transform was applied to the final lineaments extraction in the parameter space, and mapping the faults and fracture. The results showed that extraction accuracy was affected obviously by tensor voting, and the position of lineaments in the Jinglinghe Basin generally showed a similarity with previous geological map. Actually, some types of ground feature as rivers and roads may be confused with the geological elements looked for. Therefore, they are still a key error source and need further improvement to achieve higher accuracy. The orientation analysis of the lineament results revealed that the main orientations of the lineaments were NW-SE, NE-SW, NNE-SSW, and ENE-WSW, and obtained a detailed general view of tectonic stress direction successfully in this area. Despite the traces of faults are not obviously identifiable in some area, especially in flat and thick loess tableland, the integrated method still successfully in describing the discontinuities of the lineaments. Under the interaction of Liupan-Longshan Basin eastward compression and the uplift of the northern margin of the Qinling Mountains, the bedrock cutting is more obvious, while the bottom bedrock characteristics, the linear structure will be more intensive. Moreover, because of the declined Qianhe-Weihe Basin in difference and flat terrain, less lineaments were extracted from the research area in the east. The results are consistent with field geological surveys, and it is of great help to the extraction and analysis of hidden active faults. Future work will focus on the relation between lineaments and lithology units, tectonic geomorphology, and geological hazards in this area. Furthermore, the question of the linking ability of edges and the investigation of Hough peaks shape and width will also be a new insight.

\section{ACKNOWLEDGEMENTS}

This work was financially supported by the 1:50, 000 geological mapping in the loess covered region of the map sheets: Caobizhen(I48E008021), Liangting(I48E008022), Zhaoxian(I48E008023), Qianyang(I48E009021), Fengxiang(I48E009022), Yaojiagou(I48E009023) in Shaanxi Province, China, under Grant [DD-20160060]. And the project of open 
fund for key laboratory of land and resources degenerate and unused land remediation, under Grant [SXDJ2017-7].

\section{REFERENCES}

Ahmadirouhani, R., Rahimi, B., Karimpour, M. H., MalekzadehShafaroudi, A., Afshar-Najafi, S. and Pour, A. B., 2017. Fracture mapping of lineaments and recognizing their tectonic significance using spot-5 satellite data: a case study from the bajestan area, lut block, east of iran. Journal of African Earth Sciences 134, pp. 600-612.

Du, W., Chen, N. and Liu, D., 2017. Topology adaptive water boundary extraction based on a modified balloon snake: Using gf-1 satellite images as an example. Remote Sensing 9(2), pp. 140.

Duda, R. O. and Hart, P. E., 1972. Use of the hough transformation to detect lines and curves in pictures. Cacm 15(1), pp. 11-15.

Hashim, M., Ahmad, S., Johari, M. A. M. and Pour, A. B., 2013. Automatic lineament extraction in a heavily vegetated region using landsat enhanced thematic mapper (etm+) imagery. Advances in Space Research 51(5), pp. 874-890.

Koike, K., Nagano, S. and Ohmi, M., 1995. Lineament analysis of satellite images using a segment tracing algorithm (sta). Computers \& Geosciences 21(9), pp. 1091-1104.

Kusk, M. and Krbcov, K., 2015. Analysis of the relationship of automatically and manually extracted lineaments from dem and geologically mapped tectonic faults around the main ethiopian rift and the ethiopian highlands, ethiopia. Auc Geographica 52(1), pp. 5-17.

Li, J., Chen, X., Tian, L., Huang, J. and Feng, L., 2015. Improved capabilities of the chinese high-resolution remote sensing satellite gf- 1 for monitoring suspended particulate matter (spm) in inland waters: Radiometric and spatial considerations. Isprs Journal of Photogrammetry \& Remote Sensing 106, pp. 145-156.

Li, Z., Shen, H., Li, H., Xia, G., Gamba, P. and Zhang, L., 2017. Multi-feature combined cloud and cloud shadow detection in gaofen-1 wide field of view imagery. Remote Sensing of Environment 191, pp. 342-358.

Marghany, M. and Hashim, M., 2010. Lineament mapping using multispectral remote sensing satellite data. International Journal of the Physical Sciences 5(10), pp. 1501-1507.

Masoud, A. A. and Koike, K., 2011. Auto-detection and integration of tectonically significant lineaments from srtm dem and remotely-sensed geophysical data. ISPRS Journal of Photogrammetry \& Remote Sensing 66(6), pp. 818-832.

Qari, M. H. T., 2011. Lineament extraction from multi-resolution satellite imagery: a pilot study on wadi bani malik, jeddah, kingdom of saudi arabia. Arabian Journal of Geosciences 4(7-8), pp. 1363-1371.

Soto-Pinto, C., Arellano-Baeza, A. and Snchez, G., 2013. A new code for automatic detection and analysis of the lineament patterns for geophysical and geological purposes (adalgeo). Computers \& Geosciences 57(4), pp. 93-103. 